\title{
Gadolinium and nephrogenic systemic fibrosis: time to tighten practice
}

\author{
Iosif A. Mendichovszky • Stephen D. Marks • \\ Clare M. Simcock • Øystein E. Olsen
}

Received: 22 June 2007 /Revised: 31 July 2007 / Accepted: 23 August 2007 /Published online: 18 October 2007

(C) Springer-Verlag 2007

\begin{abstract}
Nephrogenic systemic fibrosis (NSF) is a relatively new entity, first described in 1997. Few cases have been reported, but the disease has high morbidity and mortality. To date it has been seen exclusively in patients with renal dysfunction. There is an emerging link with intravenous injection of gadolinium contrast agents, which has been suggested as a main triggering factor, with a lag time of days to weeks. Risk factors include the severity of renal impairment, major surgery, vascular events and other proinflammatory conditions. There is no reason to believe that children have an altered risk compared to the adult population. It is important that the paediatric radiologist acknowledges emerging information on NSF but at the same time considers the risk:benefit ratio prior to embarking on alternative investigations, as children with chronic kidney disease require high-quality diagnostic imaging.
\end{abstract}

Keywords Nephrogenic systemic fibrosis · Gadolinium . Chronic kidney disease $\cdot$ Children

\footnotetext{
I. A. Mendichovszky

Radiology and Physics Unit, Institute of Child Health, University College London,

London, UK

S. D. Marks

Department of Paediatric Nephrology,

Great Ormond Street Hospital for Children NHS Trust,

London, UK

C. M. Simcock $\cdot \varnothing$. E. Olsen $(\square)$

Department of Radiology,

Great Ormond Street Hospital for Children NHS Trust,

Great Ormond Street,

London WC1N 3JH, UK

e-mail: olseno@gosh.nhs.uk
}

\section{Introduction}

Nephrogenic systemic fibrosis (NSF; formerly named nephrogenic fibrosing dermopathy) is a systemic disease that was first described in 1997 and recognized as a separate clinical entity in 2000 [1-4]. Until now, all confirmed cases of NSF have been reported in patients with chronic kidney disease (CKD) with estimated glomerular filtration rates (eGFR) $<60 \mathrm{ml} / \mathrm{min}$ per $1.73 \mathrm{~m}^{2}$ or endstage renal failure requiring renal replacement therapy. NSF affects both sexes with a reported age range of 8-87 years and has no race predilection $[5,6]$. Relatively few paediatric cases have been reported, which may reflect the lower incidence of CKD in children.

Patients present with swelling, induration and tightening of the skin, often with a peau d'orange appearance or textured plaques, papules or nodules, usually limited to the extremities (from ankles to mid-thighs and from wrists to mid-upper arms). The skin lesions sometimes involve the trunk, but spare the neck and face. The condition develops over days to weeks and may ultimately result in severe contractures and disability. Patients may experience burning, itching or sharp pains in involved areas. Deep pain has also been described in the hips and ribs. Radiography may reveal calcifications of the soft tissue [1, 5-9]. Systemic involvement may be fatal with fibrosis of the lungs, skeletal muscles, pleura, pericardium, myocardium, kidneys, muscle, bone, testes and dura $[2-4,10]$. Clinical differential diagnoses include scleroderma, scleromyxoedema, systemic sclerosis/morphoea, eosinophilic fasciitis and eosinophiliamyalgia syndrome, and the condition may mimic cellulitis, panniculitis or drug reactions $[6-8,11,12]$.

The severity of CKD appears to be the main determinant of risk. Also, several contributing risk factors have been 
suggested: hypercoagulability and thrombotic events; cardiomyopathy; hepatic disease; idiopathic pulmonary fibrosis; systemic lupus erythematosus; brain tumours; recent surgical procedures, especially those with a major vascular component; and other proinflammatory conditions, including liver transplantation $[5,6,8,13,14]$.

A specific aetiology is still unknown. The only common factor in all patients is the underlying kidney disease, with most patients dialysis-dependent. However, NSF has also been reported in patients who have never been dialysed [1, $5,6,8]$. The physiopathological mechanism for fibrosis is not yet known. Histopathologically, biopsies from the skin lesions show a proliferation of fibrocytes (which may extend into subcutaneous tissue), together with thick collagen bundles and surrounding clefts with a variable amount of mucin and elastic fibres. Fibrocytes have a specific immunophenotype and express CD34, CD11b, CD45, HLA-DR, CD71, CD80, CD86, which implies that they are recruited from the circulation and do not arise as a result of proliferation in situ. Inflammatory cells are not usually evident, although small clusters of perivascular mononuclear cells may be seen $[11,15,16]$.

Therapeutic options in NSF are limited. Improvements have been reported with extracorporeal photopheresis, plasmapheresis, ultraviolet phototherapy, intravenous immunoglobulin and renal transplantation [17-21].

\section{Link with gadolinium-based contrast agents}

The main concern for the radiologist is a growing body of evidence that clinical use of gadolinium-based contrast agents may trigger the disease. A number of patients with NSF seem not to have had any previous gadolinium exposure, but these are still under investigation. Grobner [22] was the first to report this association in January 2006. Grobner described nine patients with end-stage renal failure on dialysis who underwent MR angiography (MRA) with gadodiamide (Omniscan; GE Healthcare, Chalfont St Giles, UK); five (mean age 57.2 \pm 10.7 years) developed NSF after 2-4 weeks. All patients who developed NSF had confirmed metabolic acidosis, whilst all patients who did not develop NSF had normal $\mathrm{pH}$ and bicarbonate level at the time of the MRA. The average contrast agent volume used was $35 \mathrm{ml}$.

Marckmann et al. [23] reviewed case notes from their own nephrology department from August 2005 to May 2006 and found 13 patients with confirmed NSF, all exposed to gadodiamide. All patients were adults (3366 years of age, mean 50 years), developed NSF 2-75 days (mean 25 days) after gadolinium exposure and had an eGFR $<8 \mathrm{ml} / \mathrm{min}$. From this cohort of renal patients the authors estimated an odds ratio between 32.5 and 47.6 for developing NSF following gadolinium exposure.
The link between gadolinium exposure and NSF has been further strengthened by two reports of gadolinium deposits in tissue samples of patients with NSF. High et al. [24] detected gadolinium in 4 out of 13 skin and soft-tissue biopsies from seven patients with documented NSF. All patients with NSF received gadolinium-based contrast medium prior to disease and the interval between dosing and biopsy could be determined with certainty only for two of four patients (age range 4-11 months). The same group reported in a research letter following quantitative analysis of the four tissue samples in which gadolinium was detected that the amount of gadolinium in the affected tissue of patients with NSF was approximately 35- to 150fold the level of gadolinium in the bone of healthy volunteers with normal renal function. In a case report, Boyd et al. [25] found gadolinium deposits in areas of calcium phosphate deposition in blood vessels in a 68-yearold patient with NSF who had been previously exposed to gadodiamide.

Sadowski et al. [26] retrospectively reviewed data from 13 patients with confirmed NSF (eight males, five females, age 17-69 years), all exposed to gadodiamide. The median time between onset of NSF symptoms and last contrastenhanced MRI examination was 11.5 days. The dose of contrast agent administered varied from 0.1 to $0.31 \mathrm{mmol} / \mathrm{kg}$ (actual) body weight. At the time of the contrast-enhanced MRI all 13 patients had CKD (eGFR $<60 \mathrm{ml} / \mathrm{min}$ per $1.73 \mathrm{~m}^{2}$ ) and were hospitalized for a proinflammatory event (major surgery, infection or vascular event). The group with NSF had significantly lower eGFR, more proinflammatory events, and more contrast-enhanced MR examinations per patient $(P<0.002)$ than did the control group. The incidence of NSF in this study and institution was $4.6 \%$ per year.

A review of six further NSF cases that were also related in time to gadodiamide administration has recently been published [27]. Patients were aged 23-71 years and the onset of symptoms consistent with NSF was between 19 days and 2 months after gadodiamide exposure. All patients had severe renal failure and were exposed to a gadodiamide dose ranging from 0.11 to $0.36 \mathrm{mmol} / \mathrm{kg}$ body weight. Despite having normal serum bicarbonate, five of the six patients had an elevated anion gap metabolic acidosis.

Broome et al. [28] have recently reported a retrospective study of 12 patients with NSF, all of whom received a double dose $(0.2 \mathrm{mmol} / \mathrm{kg}$ body weight $)$ of gadodiamide 2 11 weeks before the development of skin fibrosis. This group included eight men and four women (age 2664 years) and all had renal insufficiency at the time of gadodiamide administration. The odds ratio for development of NSF in the gadodiamide-exposed patients compared with the gadodiamide-unexposed patients was 22.3 
and the prevalence of NSF among gadodiamide-exposed dialysis patients was $4.0 \%$. The odds ratio for development of NSF with double-dose compared to single-dose gadodiamide administration was 12.1. Ten patients were dialysed within 2 days of gadodiamide administration, but this failed to prevent the development of NSF.

Although the overwhelming majority (around 90\%) of patients with gadolinium-associated NSF had been exposed to gadodiamide, there are now several reports of exposure to other gadolinium contrast agents. In a recent editorial in Radiology, Kuo et al. [29] reported 57 patients with NSF investigated by the United States Food and Drug Administration (FDA). In 43 of these the NSF was linked to gadodiamide; in 6 to gadopentetate dimeglumine (Magnevist; Berlex Imaging, Montville, N.J.); in 2 to gadoversetamide (OptiMARK; Mallinckrodt, St. Louis, Mo.); and in 3 to gadodiamide plus gadoversetamide; in 3 other patients, the associated specific gadolinium-based MR contrast agent was not definitively identified. Sadowski et al. [26] also reported one patient with confirmed NSF associated with both gadodiamide and gadobenate dimeglumine (MultiHance, Bracco Diagnostics, Milan, Italy). NSF related to specific gadolinium-based contrast agents is also monitored by the manufacturers, who have recently published several reports [30-32]. In a retrospective study in Scotland of 1,826 haemodialysis patients, Collidge et al. [33] found a positive association between the cumulative dose of gadodiamide and the development of NSF. Patients who developed NSF had received a higher median cumulative dose of gadodiamide $(0.39 \mathrm{mmol} / \mathrm{kg}$ vs. $0.23 \mathrm{mmol} / \mathrm{kg}$ in patients without NSF).

A case-control study by Marckmann et al. [34] conducted in 19 patients with histologically verified NSF and 19 matched controls found that increasing cumulative gadodiamide exposure, high-dose epoietin-b treatment and higher serum concentrations of ionized calcium and phosphate increase the risk of gadodiamide-related NSF in patients with renal failure.

\section{Pathophysiology}

Although not completely understood, several factors are known to contribute to the physiopathological mechanism in NSF. Gadolinium chelates are mostly eliminated through the kidneys. Renal impairment will therefore prolong the presence of these agents in the body, facilitating the release of the toxic free gadolinium ion $\left(\mathrm{Gd}^{3+}\right)$ by dissociation from its chelate (chemical imbalance, acidosis, anion gap, inflammation) and its subsequent binding to endogenous ions. This chemical process, known as transmetallation, may be promoted by acidosis and anion gap, e.g. under inflammatory conditions [35-40]. It has therefore been suggested that both the chemical stability of a particular chelate (cyclic compounds are more stable than linear ones in vitro) and the dose administered are risk factors for triggering NSF [28, 41]. Once released $\mathrm{Gd}^{3+}$ forms hydroxides and phosphates (insoluble at a $\mathrm{pH}>6.2$ ) and these are probably engulfed by phagocytic cells [42]. This might affect the reticuloendothelial system, inhibiting the activity of certain enzymes with consequent foreign body and fibrous reactions [41, 43-45]. Gadodiamide, the agent involved in NSF in most patients to date, is the least stable in terms of susceptibility to transmetallation in vitro, being an open-chain compound [46]. Free gadolinium ions may remain in the body for weeks to months, allowing more time for toxic effects [47]. Gadolinium is a well-known inorganic calcium channel blocker and its acute toxicity may also partly be explained via this mechanism [48, 49]. A review of the properties of commercial gadolinium contrast agents and their relation to NSF is presented in Table 1.

\section{NSF in children}

NSF in children has also been reported. Five patients under 18 years of age have thus far been reported (discussed below) out of a total of ten in the central database of the International Center for Nephrogenic Fibrosing Dermopathy Research (Dr. S. Cowper, personal communication). However, 13 possible patients have been reported to an online paediatric nephrology forum in June 2007 (https://listhost. uchicago.edu/mailman/private/pedneph/) and these are still being investigated in relation to previous gadolinium exposure.

In 2003 Jan et al. [50] reported two paediatric patients with nephrogenic fibrosing dermopathy: a 16-year-old female patient with a life-long history of kidney failure and an 8-year-old boy with a 9-month history of membranoproliferative glomerulonephritis type II. Both patients had the clinical and histopathological features of NSF and no contrast-enhanced MRI procedures were known to be associated with these patients. However, this was before the publication of a possible link with gadolinium.

Jain et al. [51] reported two more patients with NSF in 2004: a 9-year-old boy on peritoneal dialysis and a 19-yearold male on haemodialysis with a 10-year history of previous failed renal transplants, both diagnosed with NSF on skin biopsies. The 9-year-old boy had a solitary MRA performed in June 2002, approximately 2 months prior to development of NFD. The history of the 19-year-old male was more complicated as he underwent 13 separate MRA procedures, all with gadolinium contrast agents, between April 2000 and August 2004. At least nine of the MRAs were performed prior to the NFD diagnosis in January 2003 [52]. Also, both patients had persistent metabolic acidosis 


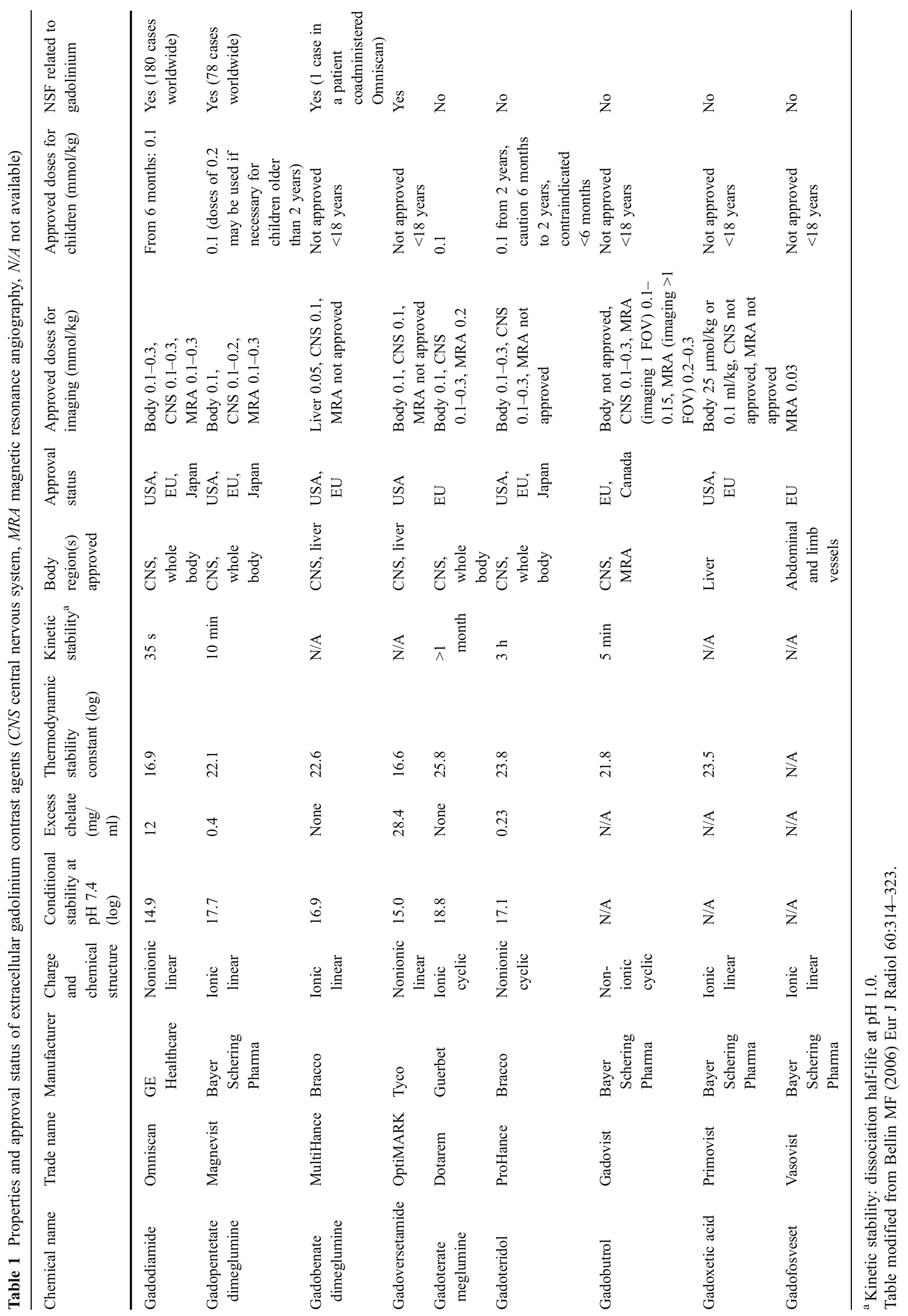


in the months preceding the development of NSF, which may have been a contributing factor [22].

DiCarlo et al. [53] described another patient with histopathologically proven NSF. This patient was a 17year-old male renal transplant recipient (15 years following transplant) who had recently begun peritoneal dialysis due to graft failure. No relationship to MRI investigations was mentioned.

Recently, Auron et al. [54] reported two patients with NSF. One of the patients was a 13-year-old boy previously reported at the age of 8 years by Jan et al. [50] in 2003 (discussed above). The second patient was a 20 -year-old male who at the age 14 years was diagnosed with acute monoblastic leukaemia and 2 years later underwent bone marrow transplant and later developed kidney failure. No references to contrast-enhanced MRI are available from these reports.

Our own hospital has a large paediatric renal unit with up to 30 renal transplants per year. We have actively used MRI both for concurrent conditions and complications to $\mathrm{CKD}$, as well as for providing a presurgical vascular roadmap, using contrast-enhanced MRA (Gd-DTPA, Magnevist, Schering, Germany, $0.1-0.3 \mathrm{mmol} / \mathrm{kg}$, i.v.). A review of all contrast-enhanced scans in children referred via our nephrology service (March 2002 to March 2007) showed that 75 nephrology patients (neonate to 18 years of age; median 9.6 years) had had 93 MRI scans with a follow-up of at least 6 months. There were no patients with NSF in this high-risk cohort.

\section{Regulatory advice}

Regulatory advice can be obtained from the websites listed in Table 2. The FDA has issued a general warning for all gadolinium-based contrast agents and advise against their use in patients with eGFR $<30 \mathrm{ml} / \mathrm{min}$ per $1.73 \mathrm{~m}^{2}$ and in all patients with acute renal insufficiency "unless the diagnostic information is essential and not available with non-contrast enhanced magnetic resonance imaging". At the FDA's request the manufacturers of gadolinium-based contrast agents have revised the product labels, which now include a new boxed warning and a new warnings section that describes the risk of NSF. The European Medicines Agency has so far not published any update of general contraindications, and is not in a position to do so since all but one gadolinium-based agent are licensed by individual member states' regulatory bodies. However, the issue was discussed at a meeting of its pharmacovigilance group in June and May 2007, and issued specific advice as follows (http://www.mhra.gov.uk):

The use of Omniscan ${ }^{\circledR}$ and Magnevist ${ }^{\circledR}$ is contraindicated in patients with severe renal impairment (i.e. GFR or eGFR $<30 \mathrm{~mL} / \mathrm{min} / 1.73 \mathrm{~m}^{2}$ ). Omniscan is also contraindicated in patients with renal dysfunction who have had, or who are awaiting, liver transplantation. For patients with moderate renal impairment (GFR or eGFR $30-59 \mathrm{ml} / \mathrm{min} / 1.73 \mathrm{~m}^{2}$ ) or neonates and infants up to 1 year of age, Omniscan and Magnevist should be used only after careful consideration. All patients should be screened for renal dysfunction by obtaining a history and/or laboratory tests, especially before Omniscan or Magnevist are used. Haemodialysis shortly after administration of a gadolinium-containing MRI contrast agent in patients currently under haemodialysis may be useful for removal of contrast agent from the body. However, there is no evidence to suggest that haemodialysis can prevent or treat development of NSF. Careful consideration should also be given to the use of the other gadolinium-containing MRI contrast agents in patients with severe renal impairment.

\section{Consequences for paediatric practice}

There is an associated high morbidity and mortality with $\mathrm{CKD}$, and imaging aids clinical management, such as assessment of secondary vasculopathies, infections etc, and surgical planning prior to renal transplantation. Patients at risk also include children with known or suspected CKD, as well as newborns and infants with renal immaturity or

Table 2 Websites for regulatory updates and for information and registration of cases (accessed on 20 September 2007)

\begin{tabular}{|c|c|c|}
\hline Organization & Information provided & URL \\
\hline European Medicines Agency & Regulatory information & http://www.emea.europa.eu/ \\
\hline \multirow[t]{2}{*}{ US Food and Drug Administration } & Regulatory information & $\begin{array}{l}\text { http://www.fda.gov/cder/drug/infopage/ } \\
\text { gcca/default.htm }\end{array}$ \\
\hline & Information for healthcare professionals & $\begin{array}{l}\text { http://www.fda.gov/cder/drug/InfoSheets/ } \\
\text { HCP/gcca 200705.htm }\end{array}$ \\
\hline $\begin{array}{l}\text { International Center for Nephrogenic Fibrosing } \\
\text { Dermopathy Research }\end{array}$ & $\begin{array}{l}\text { Updated information and contact details for } \\
\text { registration of cases }\end{array}$ & http://www.icnfdr.org/ \\
\hline
\end{tabular}


congenital cardiopathy. It seems plausible that impaired excretion, dissociation of the gadolinium chelate, and prolonged tissue retention of gadolinium is a trigger for NSF in patients with CKD. This risk increases with cumulative dose of gadolinium, with decreasing GFR $<60 \mathrm{ml} / \mathrm{min}$ per $1.73 \mathrm{~m}^{2}$, and in the presence of acidosis and proinflammatory conditions. The scarcity of reported children with NSF may well be due to the lower prevalence of CKD and secondary conditions in children. It is therefore imperative that paediatric practitioners are prudent and immediately tighten their local policies for use of intravenous gadolinium-based contrast agents.

It is difficult at present to suggest general guidelines, because:

- The absolute risk of gadolinium exposure, as well as the risk associated with the suspected contributing clinical and biochemical factors, is unknown. Each case must therefore be assessed individually.

- The risk attributable to the kinetic stability of different gadolinium-based agents is not clear. The majority of cases have been reported in association with a less stable agent (gadodiamide); however, we are still in an early stage of recording cases, and also the market share and the actual use of different agents in this patient population is not fully known. Thus, it seems reasonable to discontinue the use of gadodiamide in atrisk patients.

- The relationship to dose is not completely known. Since there is no indication that we are dealing with a side effect not related to dose, it seems reasonable to reduce the dose of gadolinium if possible, and also to monitor the cumulative dose in at-risk patients.

- It is unclear whether acidosis correction before gadolinium administration and haemodialysis immediately after gadolinium administration can prevent the development of, or treat, NSF, but such actions should be considered in children already on haemodialysis. This underlines the necessity for good communication between radiologists and nephrologists, both for issuing local guidelines and for management of the individual child.

It is often the case that MRI is requested after an inconclusive US examination. In our own experience, more meticulous patient preparation and dedicated rescanning of the patient with US can often give diagnostically adequate results. In patients with CKD not yet on dialysis CT is not a desirable alternative due to ionizing radiation and the nephrotoxicity of iodinated contrast agents. However, CT may provide an option in patients who are already on dialysis. MRI without intravenous contrast agent administration has not yet been validated for vascular anatomy prior to renal transplantation, but several techniques may be valuable, such as 3-D-balanced steady-state free precession imaging and time-of-flight angiography.

Patients with renal impairment may be referred for MRI from non-nephrology units and a crucial question is how to identify them. Our approach would be to include a mandatory tick box on the referral form requiring the referring clinician's statement about renal function, and also to include a question about any known renal problems on the patient/guardian prescan checklist. This is a pragmatic approach, which will need revision after an initial phase.

\section{Conclusion}

We would advocate prudence, and in practice suggest:

- Information collection from clinicians and patients/ guardians on renal dysfunction and active collaboration with the local nephrology unit.

- A case-by-case assessment in terms of the necessity for gadolinium-enhanced MRI, and whether an alternative test (repeat US, noncontrast-enhanced MRI, CT) would be acceptable.

- Cumulative dose recording, reduction of the dose of any gadolinium-based contrast agent, and acting accordingly to guidance published in the literature.

- Longer follow-up, especially in high-risk children.

- GFR measurement or estimation should be part of a systematic prescan investigation in children with known or suspected renal dysfunction; however, a general screening is probably not justified.

- To minimize the risks, we should take advantage of the current knowledge, and would suggest using cyclic nonionic compounds as they are more stable in vitro and therefore theoretically less likely to undergo transmetallation.

\section{References}

1. Cowper SE, Robin HS, Steinberg SM et al (2000) Scleromyxoedema-like cutaneous diseases in renal-dialysis patients. Lancet 356:1000-1001

2. Ting WW, Stone MS, Madison KC et al (2003) Nephrogenic fibrosing dermopathy with systemic involvement. Arch Dermatol 139:903-906

3. Jimenez SA, Artlett CM, Sandorfi N et al (2004) Dialysisassociated systemic fibrosis (nephrogenic fibrosing dermopathy): study of inflammatory cells and transforming growth factor beta 1 expression in affected skin. Arthritis Rheum 50:2660-2666

4. Kucher C, Steere J, Elenitsas R et al (2006) Nephrogenic fibrosing dermopathy/nephrogenic systemic fibrosis with diaphragmatic involvement in a patient with respiratory failure. J Am Acad Dermatol 54:S31-S34 
5. Cowper SE (2001-2007) Nephrogenic Fibrosing Dermopathy (NFD/NSF Website). http://www.icnfdr.org/. Cited 20 September 2007

6. Cowper SE (2003) Nephrogenic fibrosing dermopathy: the first 6 years. Curr Opin Rheumatol 15:785-790

7. Mackay-Wiggan JM, Cohen DJ, Hardy MA et al (2003) Nephrogenic fibrosing dermopathy (scleromyxedema-like illness of renal disease). J Am Acad Dermatol 48:55-60

8. Swartz RD, Crofford LJ, Phan SH et al (2003) Nephrogenic fibrosing dermopathy: a novel cutaneous fibrosing disorder in patients with renal failure. Am J Med 114:563-572

9. Cowper SE (2005) Nephrogenic systemic fibrosis: the nosological and conceptual evolution of nephrogenic fibrosing dermopathy. Am J Kidney Dis 46:763-765

10. Gibson SE, Farver CF, Prayson RA (2006) Multiorgan involvement in nephrogenic fibrosing dermopathy: an autopsy case and review of the literature. Arch Pathol Lab Med 130:209-212

11. Neudecker BA, Stern R, Mark LA et al (2005) Scleromyxedemalike lesions of patients in renal failure contain hyaluronan: a possible pathophysiological mechanism. J Cutan Pathol 32:612615

12. Solomon GJ, Wu E, Rosen PP (2007) Nephrogenic systemic fibrosis mimicking inflammatory breast carcinoma. Arch Pathol Lab Med 131:145-148

13. Obermoser G, Emberger M, Wieser M et al (2004) Nephrogenic fibrosing dermopathy in two patients with systemic lupus erythematosus. Lupus 13:609-612

14. Maloo M, Abt P, Kashyap R et al (2006) Nephrogenic systemic fibrosis among liver transplant recipients: a single institution experience and topic update. Am J Transplant 6:2212-2217

15. Cowper SE, Bucala R (2003) Nephrogenic fibrosing dermopathy: suspect identified, motive unclear. Am J Dermatopathol 25:358

16. Quan TE, Cowper SE, Bucala R (2006) The role of circulating fibrocytes in fibrosis. Curr Rheumatol Rep 8:145-150

17. Lauchli S, Zortea-Caflisch C, Nestle FO et al (2004) Nephrogenic fibrosing dermopathy treated with extracorporeal photopheresis. Dermatology 208:278-280

18. Gilliet M, Cozzio A, Burg G et al (2005) Successful treatment of three cases of nephrogenic fibrosing dermopathy with extracorporeal photopheresis. Br J Dermatol 152:531-536

19. Baron PW, Cantos K, Hillebrand DJ et al (2003) Nephrogenic fibrosing dermopathy after liver transplantation successfully treated with plasmapheresis. Am J Dermatopathol 25:204-209

20. Kafi R, Fisher GJ, Quan T et al (2004) UV-A1 phototherapy improves nephrogenic fibrosing dermopathy. Arch Dermatol 140:1322-1324

21. Chung HJ, Chung KY (2004) Nephrogenic fibrosing dermopathy: response to high-dose intravenous immunoglobulin. Br J Dermatol 150:596-597

22. Grobner T (2006) Gadolinium - a specific trigger for the development of nephrogenic fibrosing dermopathy and nephrogenic systemic fibrosis? Nephrol Dial Transplant 21:1104-1108

23. Marckmann P, Skov L, Rossen K et al (2006) Nephrogenic systemic fibrosis: suspected causative role of gadodiamide used for contrast-enhanced magnetic resonance imaging. J Am Soc Nephrol 17:2359-2362

24. High WA, Ayers RA, Chandler J et al (2007) Gadolinium is detectable within the tissue of patients with nephrogenic systemic fibrosis. J Am Acad Dermatol 56:21-26

25. Boyd AS, Zic JA, Abraham JL (2007) Gadolinium deposition in nephrogenic fibrosing dermopathy. J Am Acad Dermatol 56:2730

26. Sadowski EA, Bennett LK, Chan MR et al (2007) Nephrogenic systemic fibrosis: risk factors and incidence estimation. Radiology 243:148-157
27. Khurana A, Runge VM, Narayanan M et al (2007) Nephrogenic systemic fibrosis: a review of 6 cases temporally related to gadodiamide injection (Omniscan). Invest Radiol 42:139-145

28. Broome DR, Girguis MS, Baron PW et al (2007) Gadodiamideassociated nephrogenic systemic fibrosis: why radiologists should be concerned. AJR 188:586-592

29. Kuo PH, Kanal E, Abu-Alfa AK et al (2007) Gadolinium-based MR contrast agents and nephrogenic systemic fibrosis. Radiology 242:647-649

30. GE Healthcare (2007) Omniscan safety update. http://www. amershamhealth-us.com/omniscan/. Cited 20 September 2007

31. Bayer Healthcare Pharmaceuticals (2007) Magnevist labeling update, May 23, 2007. http://www.imaging.bayerhealthcare.com/ html/magnevist/labeling_update.html. Cited 20 September 2007

32. Leiner T, Herborn CU, Goyen M (2007) Nephrogenic systemic fibrosis is not exclusively associated with gadodiamide. Eur Radiol 17:1921-1923

33. Collidge TA, Thomson PC, Mark PB et al (2007)Gadoliniumenhanced MR imaging and nephrogenic systemic fibrosis: retrospective study of a renal replacement therapy cohort. Radiology. DOI 2451070353

34. Marckmann P, Skov L, Rossen K et al (2007) Case-control study of gadodiamide-related nephrogenic systemic fibrosis. Nephrol Dial Transplant. DOI 10.1093/ndt/gfm261

35. Joffe P, Thomsen HS, Meusel M (1998) Pharmacokinetics of gadodiamide injection in patients with severe renal insufficiency and patients undergoing hemodialysis or continuous ambulatory peritoneal dialysis. Acad Radiol 5:491-502

36. Idee JM, Berthommier C, Goulas V et al (1998) Haemodynamic effects of macrocyclic and linear gadolinium chelates in rats: role of calcium and transmetallation. Biometals 11:113-123

37. Corot C, Idee JM, Hentsch AM et al (1998) Structure-activity relationship of macrocyclic and linear gadolinium chelates: investigation of transmetallation effect on the zinc-dependent metallopeptidase angiotensin-converting enzyme. J Magn Reson Imaging 8:695-702

38. Behra-Miellet J, Gressier B, Brunet C et al (1996) Free gadolinium and gadodiamide, a gadolinium chelate used in magnetic resonance imaging: evaluation of their in vitro effects on human neutrophil viability. Methods Find Exp Clin Pharmacol 18:437442

39. Green RW, Krestin GP (2006) Non-tissue specific extra cellular MR contrast media. In: Thomsen HS (ed) Contrast media: safety issues and ESUR guidelines. Springer, Berlin, pp 107-112

40. Idee JM, Port M, Raynal I et al (2006) Clinical and biological consequences of transmetallation induced by contrast agents for magnetic resonance imaging: a review. Fundam Clin Pharmacol 20:563-576

41. Harpur ES, Worah D, Hals PA et al (1993) Preclinical safety assessment and pharmacokinetics of gadodiamide injection, a new magnetic resonance imaging contrast agent. Invest Radiol 28 [Suppl 1]:S28-S43

42. Spencer AJ, Wilson SA, Batchelor J et al (1997) Gadolinium chloride toxicity in the rat. Toxicol Pathol 25:245-255

43. Mendoza FA, Artlett CM, Sandorfi N et al (2006) Description of 12 cases of nephrogenic fibrosing dermopathy and review of the literature. Semin Arthritis Rheum 35:238-249

44. Itoh N, Kawakita M (1984) Characterization of Gd3+ and Tb3+ binding sites on $\mathrm{Ca} 2+, \mathrm{Mg} 2+-$ adenosine triphosphatase of sarcoplasmic reticulum. J Biochem (Tokyo) 95:661-669

45. Husztik E, Lazar G, Parducz A (1980) Electron microscopic study of Kupffer-cell phagocytosis blockade induced by gadolinium chloride. Br J Exp Pathol 61:624-630

46. Puttagunta NR, Gibby WA, Puttagunta VL (1996) Comparative transmetallation kinetics and thermodynamic stability of gadolin- 
ium-DTPA bis-glucosamide and other magnetic resonance imaging contrast media. Invest Radiol 31:619-624

47. Bartolini ME, Pekar J, Chettle DR et al (2003) An investigation of the toxicity of gadolinium based MRI contrast agents using neutron activation analysis. Magn Reson Imaging 21: 541-544

48. Krasnow N (1977) Lanthanide binding to cardiac and skeletal muscle microsomes. Effects of adenosine triphosphate, cations, and ionophores. Arch Biochem Biophys 181:322-330

49. Lansman JB (1990) Blockade of current through single calcium channels by trivalent lanthanide cations. Effect of ionic radius on the rates of ion entry and exit. J Gen Physiol 95:679-696
50. Jan F, Segal JM, Dyer J et al (2003) Nephrogenic fibrosing dermopathy: two pediatric cases. J Pediatr 143:678-681

51. Jain SM, Wesson S, Hassanein A et al (2004) Nephrogenic fibrosing dermopathy in pediatric patients. Pediatr Nephrol 19:467-470

52. Dharnidharka VR, Wesson SK, Fennell RS (2007) Gadolinium and nephrogenic fibrosing dermopathy in pediatric patients. Pediatr Nephrol 22:1395

53. DiCarlo JB, Gupta EA, Solomon AR (2006) A pediatric case of nephrogenic fibrosing dermopathy: improvement after combination therapy. J Am Acad Dermatol 54:914-916

54. Auron A, Shao L, Warady BA (2006) Nephrogenic fibrosing dermopathy in children. Pediatr Nephrol 21:1307-1311 